\title{
SUMÁRIO/ CONTENTS / CONTENIDO
}

\section{EDITORIAL}

Os Desafios para 2015

The Challenges to 2015

Los Desafíos para 2015

Álvaro da Silva Santos

\section{ARTIGOS /ARTICLES /ARTÍCULOS}

Uso da Prótese Dentária entre Idosos: um problema social.

Use of Dental Prosthesis among Elderly: a social problem

Uso de Prótesis Dental entre Ancianos: un problema social

Mário Alfredo Silveira Miranzi, Maristela Marques Amuí, Helena Hemiko Iwamoto, Darlene Mara dos Santos

Tavares, Sandra Azevedo Pinheiro, Marli Aparecida Reis Coimbra

A Prática Educativa no Ensino Fundamental.

Educational Practice in the Primary Education

La Práctica Educativa en la Enseñanza Primaria

Jussara Gabriel dos Santos

Família, Desenvolvimento Bioecológico e Adoecimento Mental

Family, Bio-ecological Development and Mental Illness

Familia, Desarrollo Bioecologico y la Enfermedad Mental

Dennis Gabiatti Lopes, Eduardo Sousa Gotti, Lúcio Andrade Silva, Maria Carolina Bizinoto Caetano, Raquel Cornélio Marin, Fabio Scorsolini-Comin

Espiritualidade, Religiosidade e Religião nas Políticas Públicas de Saúde: um olhar para a integralidade.

Spirituality, Religiosity and Religion on Public Health Policies: a look at integrality

Espiritualidad, Religiosidad y Religión en las Políticas Públicas de Salud: una mirada para la integralidad

Carolina Nantes de Castilho,Paula Tatiana Cardoso

Estágio de Pesquisa em História: relatando a experiência da digitalização de documentos oficiais.

Research Internship in History: reporting the experience of scanning official documents

La Pasantía de Investigación en Historia: relatando la experiencia de la digitalización de documentos oficiales

Clayton Cardoso Romano, Larissa Stephani Costa, Rochelle Gutierrez Bazaga, Thayane da Rocha Cruz Dias Freitas, Wagner da Silva Teixeira

O Fenômeno Reacional na Hanseníase e Aspectos da Assistência de

Enfermagem

The Reactional Phenomenon in Leprosy and Aspects of Nursing Care

El Fenómeno Reaccional en Lepra y Aspectos de la Asistencia de Enfermería

Vania Del'Arco Paschoal, Zaida Aurora Sperli Geraldes Soler

Estágio Supervisionado em Serviço Social: compartilhando experiências e reflexões expressas numa universidade pública.

Supervised Internship in Social Service: sharing experiences and thoughts expressed in a public university

Pasantía Supervisada en Servicio Social: compartiendo experiencias y reflexiones expresadas en una universidad pública

Lesliane Caputi, Rita de Cássia Lopes de Oliveira Mendes 